\title{
Determination of transformer oil quality by the acoustic method
}

\author{
Anastasiya Tyuryumina*, Andrey Batrak and Victor Sekackiy \\ Siberian Federal University, 26 Kirenskiy st., Krasnoyarsk, 660074, Russian Federation
}

\begin{abstract}
Large power transformers are the most expensive and strategically important components of any power generation and transmission system. Their reliability has crucial importance for the energy system operation. Insulation breakdown can generate a serious failure of a large power transformer, which causes substantial costs to repair and financial loss due to power outage. Most power utilities are, therefore, highly motivated to assess the actual condition of the transformer insulation system. The majority of transformer condition check methods are only available for off-line periodic diagnostics. The acoustic emission (AE) method as a complex online diagnostics technique to identify evolving failures is proposed. Results of measuring the acoustic signals generated by the presence of impurities in transformer oil and determining the impurities influence on its condition as an indicator of the transformer condition is presented.
\end{abstract}

\section{Introduction}

The power transformers are the most important and expensive part in a power system and considered to be reliable components of electrical stations if their design life is $20-35$ years (increasing to 50 years in practice with appropriate maintenance). Effective condition monitoring of these assets can protect loss of supplies to large populations of customers, while the threat of explosions and fire, causing irreparable damage, can injury the station and personnel and pose a risk to safety and the environment with potentially serious legal and regulatory implications. Therefore, the welfare of transformers remains at the forefront of utilities' asset management and obligations [1].

A survey of senior utility managers [2] showed that more than two-thirds of answers were related to the adverse stock of transformers, an issue which was one of the most important problems for utilities. The majority of power transformers currently in operation were installed in the 60's and 70's of the previous century. They might have reached a critical age with an operating time of 30 years and more. Based on obtained answers, Kurtz et al. [2] determined that the amount of problematic units is maximal when transformers reach 35-40 years (Fig. 1).

Utilities have to perform modernization or replacement of service-aged transformers, depending on each transformer technical condition and operational costs. A modernized transformer may serve 10-15 years but requires a careful maintenance, regular inspections and checks. Therefore, the investment in a new transformer may be postponed. The modernization costs are significantly less than a new unit cost [3].

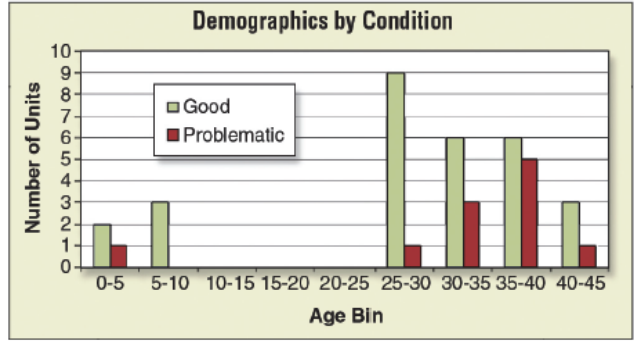

Fig. 1. Typical demographic data sorted by condition [2].

Malewski et al. [3] calculated the costs of a typical transformer. The cost of components and labor involved in modernization was $23 \%$ of a new transformer cost. At the same time the cost of condition assessment $(1 \%)$ was negligible compared to the modernization cost. It was especially relevant for high power transformers because their investment cost in relation to the total cost of transmission, division and distribution devices was about $20 \%$. In extreme cases transformer failure cost might exceed its construction cost five times [4].

Therefore, EAM is an important issue of operation management and should be applied to power transformer maintenance including advanced fault prevention and immediate diagnostics to avoid unexpected breakdowns. Some components of EAM systems in the majority of countries were discussed in [5-11].

Therefore, that the utility managers were unanimous in their assessment that improved methodologies are necessary for power transformers [2]. Currently, there has been a growing interest in the condition assessment of transformer insulation. Since the majority of transformer components are located within the main tank, the transformer oil sample can provide the information about its condition [12].

\footnotetext{
* Corresponding author: eternity17@list.ru
} 
Estimated lifetime of an insulation system was reduced for the half if average life time temperature of transformer increased by $8^{\circ} \mathrm{C}$ over maximum allowable operating temperature. Practically, it means that estimated lifetime of a 50 year-old transformer is reduced to 25 years if maximum allowed operating temperature $\left(90^{\circ} \mathrm{C}\right)$ is exceeded on average by $8^{\circ} \mathrm{C}[13]$.

There is variety of reasons to increase operating temperature. This study prefers to discuss water content, cellulose and gas phrase as the main reasons.

\section{Effect of impurities on transformer oil condition}

\subsection{Water influence}

The importance of moisture presence in paper-oil systems has been recognized since the 1920s [14]. Pure transformer oil consists of saturated hydrocarbons such as paraffins and naphthenes. Because of their nonpolar molecule structure, pure oil can dissolve only a very small quantity of water dispersed as water vapour according to Henry's law [15]. One drop of water is enough to saturate one liter of new oil [16].

Water causes three dangerous effects: it decreases the dielectric withstand strength, accelerates cellulose aging, and causes the emission of bubbles at high temperatures [17-20]. The knowledge about the actual moisture concentration is required to control transformer condition. Therefore, different methods and instrumentation are used to measure moisture.

Equilibrium diagram methods attempt to derive moisture in solid insulation from moisture content in oil (Du el al. [14], Fabre et al. [18], Oommen [21], Griffin et al. [22]) However, the methods fail, where aging of oil and water titration has the major impact [23].

Karl Fischer introduced the titration method in 1935. Titration basically means adding a reagent of a known concentration (titre) to an unknown substance until the concentrations are balanced. It measures moisture content related to oil weight (ppm as $\mu \mathrm{g}$ water/g oil) or as relative moisture saturation to saturation value $(\%)$.

Nowadays this method is considered to be the most reliable method and it also serves as a benchmark for other methods. Nevertheless, this method is subject to several faults, which may lead to a bad comparability of results. They are listed below:

- there is always a moisture ingress from atmosphere during sampling, transportation and sample preparation;

- cellulose binds water with chemical bonds of different strengths and releases water under the influence of heating and time;

- laboratories differently treat constraints that are not covered by standards, e.g. the solvent for oil extraction; - sometimes direct injection and the heating method lead to different results of moisture measurement due to the influence of oil additives and ageing products [15].

In addition, the Karl Fischer titration method is not a real-time one, and requires appropriate chemical instrumentation. It is also prone to a high level of error and its results are often difficult to interpret.
A group of methods have attracted a lot of attention in recent years. They rely on the measurement and analysis of insulation dielectric properties. This diagnostics is based on the fact that paper and oil change their dielectric properties when moisture levels increase. Therefore, measuring parameters can be the dissipation factor, polarization and depolarization currents (PDC) and return voltages (RVM). Also, to evaluate water content in oil, polymer relative humidity/relative saturation sensors are frequently used [16].

However, the majority of dielectric methods do not distinguish moisture impact on an insulation aging from impact of other processes. This creates a lot of confusion over the reliability of dielectric response methods.

Water in a solid insulation is known to play a role of an ageing process catalyst. However, transformer oil in power transformers is polluted with refining remains, ageing byproducts and cellulosic particles. In aged oil, water can be adsorbed by impurities, e.g. cellulose fibres, when the saturation level is exceeded and water solubility increases [15].

\subsection{Cellulose influence}

Paper used as an insulation material includes $85 \%$ of cellulose, 3-7 \% of lignin and $10 \%$ of hemicelluloses. Transformer paper ages under the influence of heat, air and moisture and eventually loses all mechanical integrity, with risk increasing dramatically due to insulation damage [24].

Fourier transform infrared (FTIR) and near-infrared (NIR) spectroscopy are used to characterize ageing of cellulose and assess paper insulation condition. They determine the differences in spectra of new and aged materials e.g. Ali et al. [25] studied aged cellulose and obtained a 0.994 correlation between the spectra and ageing time, with an error of 95 hours prediction for paper samples with up to 3000 hours of ageing.

Generally, the methods studying cellulose in transformer focus on determining its condition, although, brittle paper may break away from transformer windings and block ducts [26]. We propose to determine cellulose sediment influence on transformer oil condition with the $\mathrm{AE}$ method and use obtained information to evaluate transformer condition.

\subsection{Influence of dissolved gases}

Another detrimental effect which can occur in transformer oil is gaseous bubbles. This phenomenon results in dangerous effects, such as formation of vapour-filled cavities on insulation surface and in oil, with dramatic decrease of insulation dielectric strength; deimpregnation of the turn insulation.

The breakdown process starts with a microscopic bubble, an area of large distances between corpuscles, where ions or electrons can initiate avalanches. These microscopic bubbles originate from current impulses on an electrode. The next current impulse injects charge carriers into the bubble, leading to current amplification and finally to breakdown [27]. 
Applying method of dissolved gas analysis (DGA) and taking samples of mineral oil inside transformer, we can determine what gases are present and find out their concentration levels. Available gases from chromatographic analysis of insulation oil could contain concentrations of dissolved carbon monoxide (CO), carbon dioxide $\left(\mathrm{CO}_{2}\right)$, nitrogen $\left(\mathrm{N}_{2}\right)$, hydrogen $\left(\mathrm{H}_{2}\right)$, methane $\left(\mathrm{CH}_{4}\right)$, acetylene $\left(\mathrm{C}_{2} \mathrm{H}_{2}\right)$, ethylene $\left(\mathrm{C}_{2} \mathrm{H}_{4}\right)$, and ethane $\left(\mathrm{C}_{2} \mathrm{H}_{6}\right)$ [28].

Halstead [29] argued that there was a relationship between fault temperature and the composition of gases dissolved in oil. In [30] Griffin determined key gases and what faults these gases could identify. Zhang et al [31] established diagnostics criteria for types and amounts of gases in oil. Compositions of the dissolved gases, rates of generation, and specific content ratios can be used to indicate transformer condition.

Some artificial intelligence techniques present diagnostic schemes to analyze the dissolved gas data e.g. Huang et. al. [32], Islam et al. [33], Zhang et. al [34]. Each of these techniques can identify a single impurity in transformer oil. Therefore, applying a combination of these techniques to determine three impurities is inexpedient, requires much time, incurs financial and labour costs. We propose to use the $\mathrm{AE}$ method to determine these impurities and their influence on transformer oil condition as an indicator of transformer condition.

\section{Acoustic emission method}

The AE method has been studied for 25 years [35], in particular in Poland, Brazil and Taiwan, to determine partial discharge impact [35-38]. Various physical phenomena accompany the occurrence and development of partial discharges (PDs) in insulation systems. They are chemical changes of insulation, the occurrence of the current pulse and emission of an electromagnetic wave, and a percussive elastic strain with an acoustic wave generation [35]. To avoid these effects, the AE method can be used. It is now a significant supplement for other measuring methods used in diagnostics of transformer insulation systems, current and voltage measuring transformers, power condensers and bushing insulators.

The most significant merit of the AE method is the possibility of its application in difficult operation conditions. It enables detecting the PD occurrence tracing in power equipment operation, locating the places of PD generation in insulation systems. Therefore, the AE method fills the gap in PD metrology of highpower equipment. Procedures of AE pulses' measure and registration are discussed in [39].

We used the AE method to measure acoustic signals generated by impurities (water, cellulose, gas phrase) in transformer oil and to analyze their frequency spectrum to acquire the information about transformer condition.

\section{Materials and methods}

As the studied sample the transformer oil of T-750 and $\mathrm{T}-1500$ brands was applied, the independent variables were quantity of impurities in oil samples and generator frequency, the dependent variable was sound wave power value.

The measurement setup consisted of a transformer tank filled with electro-insulating oil and impurities, two electro-mechanical (piezoelectric) transducers, AE signal generator, a personal computer (PC) and designed software. The principal scheme of acoustic signal processing is presented in Figure 2.

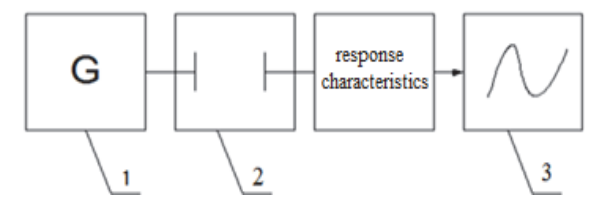

Fig. 2. Principal scheme of acoustic signal processing: 1 generator; 2 - piezoelectric transducers, 3 - measuring system.

To measure the AE pulses, a piezoelectric transducer was used, it was placed inside the tank. Its application made the measurement of sinusoidal acoustic signals possible at amplitude characteristics in the frequency range from $1 \mathrm{kHz}$ to $10 \mathrm{kHz}$ with a step from 2 to 20 $\mathrm{kHz}$ properly.

The registration of the AE pulses was done with the original software compatible with a PC and, to the best of our knowledge, new to the field. The AE pulses registered underwent the analysis in the time, frequency and time-frequency domains and were visualized with the computer program Matlab 6.0.

First, the comparison test of a new and aged transformer oils was performed to check the AE method sensitivity. The acoustic signals of both oils were studied in $1-10 \mathrm{kHz}$ frequency range at room temperature.

To determine the effect of water, cellulose, gas on transformer oil condition, we added them to different samples of new oil and measured the AE signals.

Water was added to the first transformer oil sample with a pipette. The mixture was weighed. Sound wave power was measured in 4 points, where percentage scale of water mass concentration in transformer oil was $0 \%$, $0,224 \%, 0,355 \%, 0,0994 \%$. This range was chosen in view of oil hygroscopicity. Measurement error did not exceed 0,000496 g [40-43].

Similarly, we added cellulose to the second transformer oil sample. Cellulose mass concentration in transformer oil being $0 \%, 0,06 \%, 0,18 \%, 0,31 \%$. Gas addition was performed by air input through a slotter pipe with the speed of $3,33 \mathrm{ml} / \mathrm{s}$ in a tank with oil sample [42].

\section{Results}

The comparison test showed that the AE signals measured in the samples of new and aged transformer oils were significantly different. Therefore, the content of impurities in transformer oil had an impact on sound wave power (see Fig. 3). The obtained AE signals were recorded and the response characteristics of transformer oil with impurities were represented. 


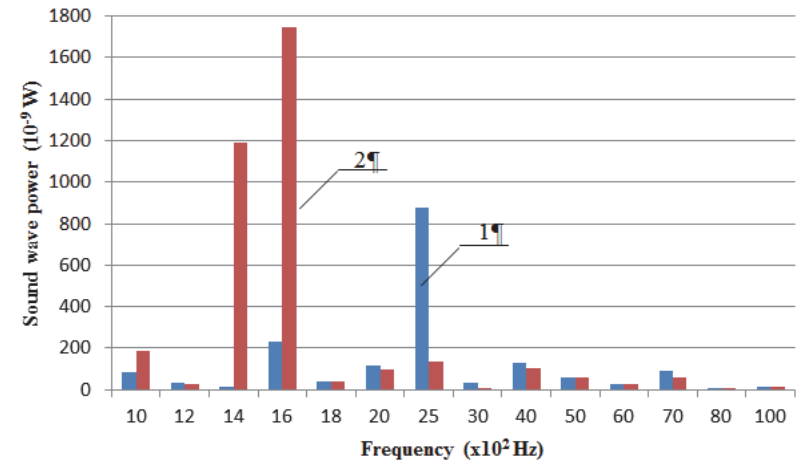

Fig. 3. Response characteristics of a new and aged transformer oils: 1 new transformer oil, 2 aged transformer oil.

The obtained sound power vs. frequency diagrams of oil samples with water, with cellulose and gas phrase were represented in Figures 4-6 respectively.

The response characteristics of model oils without impurities differed from the response characteristics of oil sample with impurities. Therefore, the presence of impurities influenced transformer oil condition.

The effect of impurities was determined applying the Fisher's test. As a result, water and cellulose influenced transformer oil quality, in contrast to gas phase. The AE method was not sensitive to determining gas phase in transformer oil in the selected the frequency range.

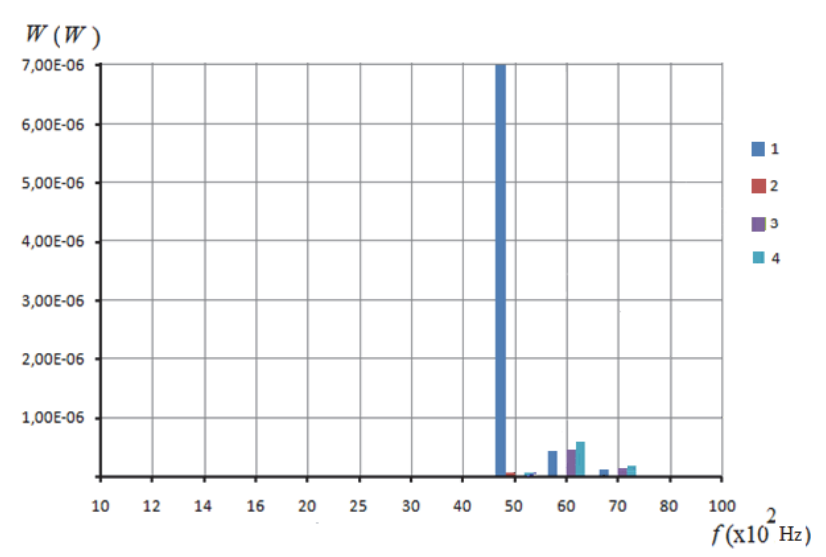

Fig. 4. Response characteristics of transformer oil with water: 1 - water $0,355 \% ; 2$ - water $0,224 \% ; 3$ - model oil (water $0 \%$ ), 4 - water $0,0994 \%$.

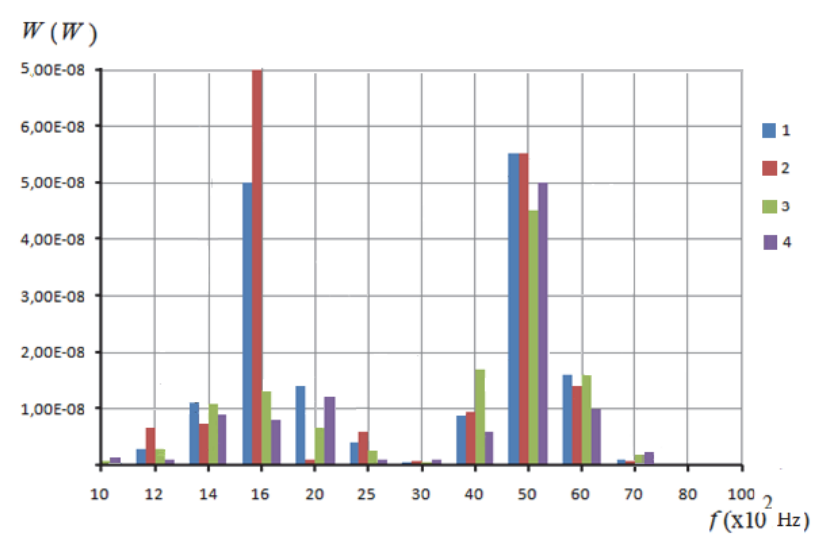

Fig. 5. Response characteristics of transformer oil with cellulose: 1 - cellulose $0,06 \% ; 2$ - cellulose $0,18 \% ; 3$ - cellulose $0,31 \%$; 4 - model oil (cellulose $0 \%$ ).

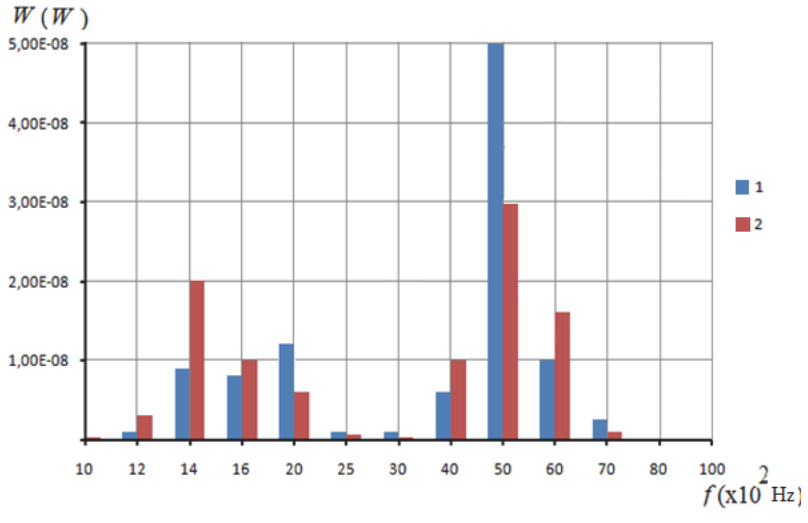

Fig. 6. Response characteristics of transformer oil with/without gas phase: 1 - gas phase presence; 2 - gas phase absence.

Applying the regression procedure to the $2 \mathrm{kHz}, 5$ $\mathrm{kHz}, 8 \mathrm{kHz}$ frequencies were studied to obtain a correlation between the sound wave power and water content in oil. The correlation coefficient values were $0,96,0,84,0,94$ for the $2 \mathrm{kHz}, 5 \mathrm{kHz}, 8 \mathrm{kHz}$ frequencies respectively. Therefore, the dependence between the sound wave power and water in the range from 0 to $0,335 \%$ was established as linear which had type:

$$
Y=b_{0}+b_{1} X
$$

Determining the regression coefficients, for the 2 $\mathrm{kHz}, 5 \mathrm{kHz}, 8 \mathrm{kHz}$ frequencies the following equations were obtained:

$$
\begin{aligned}
& Y_{2 \mathrm{kHz}}=9,94 \cdot 10^{-9}-2,76 \cdot 10^{-8} \cdot \mathrm{X} \\
& Y_{5 \mathrm{kHz}}=-4 \cdot 10^{-8}+1,22 \cdot 10^{-6} \cdot \mathrm{X} \\
& Y_{8 \mathrm{kHz}}=8,54 \cdot 10^{-10}+4,1 \cdot 10^{-9} \cdot \mathrm{X}
\end{aligned}
$$

Figure 7 represented modal and experimental dependences between sound wave power and water concentration for the $2 \mathrm{kHz}$ frequency [44].

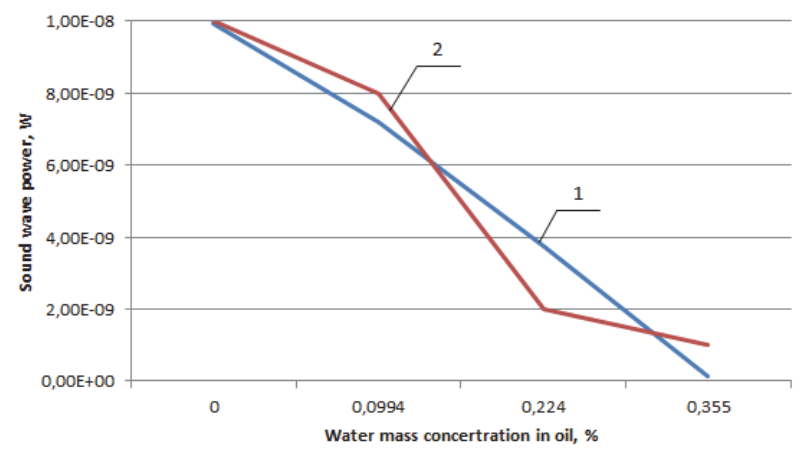

Fig. 7. Comparison of model and experimental curves of water content for $2 \mathrm{kHz}$ frequency: 1 - model dependence; 2 experimental dependence.

Similarly, the $1 \mathrm{kHz}, 1,6 \mathrm{kHz}, 5 \mathrm{kHz}$ frequencies were studied to obtain a correlation between the sound wave power and cellulose. The correlation coefficients were $0,03,0,02$ and 0,53 . Therefore, this dependence was not linear and had more sophisticated character.

Our future work may include determination of more sophisticated model of the correlation between the sound 
wave power and cellulose content in transformer oil and establishing a gas effect on transformer oil condition.

Compared to other methods, the AE method had a number of advantages. For example, its possibility to determine the content of several impurities applying the same equipment in field, e.g. in a substation or in a power station. Therefore, this method is available for complex online diagnostics. Compared to other methods it is simpler, cheaper, less time-consuming as we do not need to transport oil samples to an equipped laboratory, use sophisticated reagents, and skills.

\section{Conclusion}

Water, cellulose and gas phase were determined as the main reasons of transformer oil degradation. Various methods used to determine the impact of the impurities were presented in the paper. The AE method was highlighted as the most perspective method for online complex diagnostics of transformer oil condition. A detailed explanation of the experiments with the $\mathrm{AE}$ method was presented.

Taking into account the fact that transformer oil contains about $70 \%$ of the available diagnostics information, the obtained results can be important for the utilities which aim to reduce maintenance costs, lengthen transformer's life, increase operation safety, minimize accidental and severe failures of power transformers, and for energy consumers who aim at reliable energy supply for the low cost.

\section{References}

1. S.M. Strachan, S. Rudd, S.D.J. McArthur, M.D. Judd, S. Meijer, E. Gulski, IEEE Transactions on Dielectrics and Electrical Insulation, 1 (2008)

2. C. Kurtz, G. Ford, M. Vainberg, M. Lebow, B. Ward, Transmission \& Distribution World, July (2005)

3. R. Malewski, J. Subocz, M. Szrot, J. Plowucha, R. Zaleski, CIGRE Session 2008, Paris (2008)

4. T. Boczar, P. Fracz, Physics and chemistry of solid state, 3 (2006)

5. A. Tyuryumina, V. Sekackiy, A. Batrak, The modern materials, technics and technologies, 3(3) (2015)

6. A. Tyuryumina, A. Batrak, Issues of modern science theory and practice: records of $V$ International theoretical and practical conference (2016)

7. A. Tyuryumina, A. Batrak, V. Sekackiy, The young scientist, 8 (2016)

8. A. Tyuryumina, A. Batrak, V. Sekackiy, The young scientist, 28 (2016)

9. A. Tyuryumina, Svobodniy Avenue - 2016: proceedings of the scientific conference (2016)

10. A. Tyuryumina, Svobodniy Avenue - 2016: proceedings of the scientific conference (2016)
11. A. Tyuryumina, V. Sekackiy, A. Batrak, The complex systems in extreme conditions: proceedings of XVIII All-Russian symposium with international participation (2016)

12. P. Verma, Y.R. Sood, J. Singh, Proceedings of the $16^{\text {th }}$ International Symposium on High Voltage Engineering (2009)

13. C. Sumereder, M. Muhr, B. Körbler, $17^{\text {th }}$ International Conference on Electricity Distribution

14. Y. Du, M. Zahn, B.C. Lesieutre, A.V. Mamishev, S.R. Lindgren, Feature Article, 1 (1999)

15. M. Koch, S. Tenbohlen, J. Blennow, I. Hoehlein, $15^{\text {th }}$ ISH (2007)

16. T. Gradnik, M. Konkan-Gradnik, N. Petric, N. Muc, IEEE International Conference on Dielectric Liquids (2011)

17. F.M. Clark, Transactions of electrical engineering, 61 (1942)

18. J. Fabre, A. Pichon, International Conference on Large High Voltage Electric System (CIGRE) (1960)

19. H.P. Moser, Special print of Scientia Electrica, (EHV-Weidmann Lim., St., Johnbury, Vermont, USA, Section C, 1979)

20. W.A. Fessler, W.J. McNutt, T.O. Rouse, EPRI report, EL-5384 (1987)

21. T.V. Oommen, Electrical/Electronics Insulation Conference (1983)

22. P.G. Griffin, C.M. Bruce, J.D. Christie, Minutes of the Fifty Fifth Annual International Conference of Doble Clients (1988)

23. M. Koch, XIVth International Symposium on High Voltage Engineering (2005)

24. A.M. Emsley, G.C. Stevens, Cellulose, 1 (1994)

25. M. Ali, A.M. Emsley, H. Herman, R.J. Heywood, Polymer, 42 (2001)

26. C. Sumereder, M. Muhr, B. Körbler, $17^{\text {th }}$ International Conference on Electricity Distribution (2003)

27. M. Butcher, Mechanisms of charge conduction and breakdown in liquid dielectrics, ( $\mathrm{PhD}$ dissertation, Graduate Faculty of Texas Tech University, 2005)

28. Chia-Hung Lin, Chien-Hsien Wu, Ping-Zan Huang, Expert Systems with Applications, 36 (2009)

29. W.D. Halstead, Journal of the Institute of Petroleum, 59 (1973)

30. P.J. Griffin, STP998. American Society for Testing and Materials, 1 (1988)

31. Y. Zhang, X. Ding, Y. Liu, P.J. Griffin, IEEE Transactions on Power Delivery, 11(4) (1996)

32. Y.-C. Huang, H.-T. Yang, C.-L. Huang, IEEE Transactions on Power Delivery, 12(2) (1997)

33. S.M. Islam, T. Wu, G. Ledwich, IEEE Transactions on Dielectrics and Electrical Insulation, 7(2) (2000)

34. T. Boczar, Physics and chemistry of solid state, 3 (2007) 
35. T. Boczar, P. Fracz, Physics and chemistry of solid state, 3 (2006)

36. D. Park, S. Choi, G. Kil, The $7^{\text {th }}$ WSEAS International Conference on Power Systems (2007)

37. O.G. Santos Filho, S.L. Zaghetto, G.O. Pereira, $17^{\text {th }}$ World conference on nondestructive testing, Shanghai, China (2008)

38. D. Wotzka, T. Boczar, D. Zmarzly, Acta Physica Polonica, A 3 (2009)

39. T. Boczar, Journal of Acoustic Emission, 17(3-4) (1999)

40. A. Batrak, A. Tyuryumina, A. Nikitina, Heavy equipment industry, 3 (2013)

41. A. Batrak, A. Tyuryumina, A. Nikitina, XIII International youth scientific conference Intellect and science (2013)

42. A. Batrak, A. Tyuryumina, A. Nikitina, XIV AllRussian youth scientific conference with international participation Intellect and science (2014)

43. A. Batrak, T. Chupac, A. Tyuryumina, A. Nikitina, Heavy equipment industry, 4-5 (2014)

44. A. Batrak, A. Tyuryumina, A. Nikitina, $V$ International youth scientific conference Youth and XXI century (2015) 